\title{
Reflex modification produced by visual stimuli in the rat following functional decortication
}

\author{
JAMES R. ISON, G. PETER BOWEN, and KEVIN O'CONNOR \\ University of Rochester, Rochester, New York
}

\begin{abstract}
The effect of light prepulses on acoustic startle reflexes in rats was tested after bilateral application of $\mathrm{KCl}$ or saline solutions to the cortex, in a within-subjects design. With saline, the light maximally inhibited reflex expression at lead times of 40-70 msec, with recovery thereafter to 500 msec. In contrast, there was no inhibition with $\mathrm{KCl}$-produced decortication, but the prepulses facilitated reflex expression at lead times of $110 \mathrm{msec}$ and above. The loss of inhibition with $\mathrm{KCl}$ reveals that the cortex may directly participate in reflex inhibition produced by visual stimuli, or else must strongly influence subcortical structures essential for their effectiveness. The anomalous appearance of facilitation shows that the decorticate animal processes visual information, but in an atypical fashion. In other experiments, the inhibitory effect of noise prepulses has not been affected by cortical depression. Thus, we conclude that visual processing, not "generic inhibitory processing," is under cortical control.
\end{abstract}

Irrelevant pulsatile stimuli that are presented at certain brief intervals before the elicitation of an acoustic startle reflex inhibit reflex expression in laboratory animals and in humans. The amount of inhibition is determined in part by the intensity of the initial stimulus, and in part by its lead time, as inhibition increases to a maximum at an optimal interval and declines thereafter (Hoffman \& Ison, 1980). In the present study, we examined the potential role of cortical activity in the production of reflex inhibition by visual stimuli in the rat. The majority of the physiological studies of this phenomenon have been intended to determine the location of the responsible neural structures, and there is general agreement that reflex inhibition depends critically on the integrity of brainstem mechanisms (Leitner \& Cohen, 1985; Leitner, Powers, Stitt, \& Hoffman, 1981); that it can be simulated by brainstem stimulation (Saitoh, Tilson, Shaw, \& Dyer, 1987); and that it is unaffected by substantial damage to the forebrain (Davis \& Gendelman, 1977; Hammond, 1974; Kemble \& Ison, 1971). These studies support in detail the original findings of Sechenov (1863/1965), who showed that brainstem stimulation and brainstem ablation had similar coherent effects on reflex inhibition in the frog.

The present work reflects a related but different intent, which is, namely, to begin to identify the neural structures that are necessary in order for the various unique modalities and qualities of sensory input to gain access

\footnotetext{
This research was supported in part by a research grant from the U.S. Environmental Protection Agency (CR-806820), and in part by an anonymous donor. Analyses of the data were supported by a U.S. Public Health Service Grant EY-01319, to the Center for Visual Science at the University of Rochester. Reprint requests should be sent to James R. Ison, Department of Psychology, Meliora Hall, University of Rochester, Rochester, NY 14627.
}

to the inhibitory brainstem structures. For acoustic pulses, for example, it appears that peripheral and brainstem auditory structures provide not only the necessary, but also the sufficient neural substrate for reflex inhibition (Leitner \& Cohen, 1985; Young \& Fechter, 1983), in that, as described previously, their influence survives drastic interference with the forebrain. In contrast, however, the inhibitory effects of brief gaps in noise, rather than noise pulses, or even noise offsets, require the additional presence of intact cortical functioning (Ison, O'Connor, Bowen, \& Bocirnea, 1991), perhaps because components of the auditory cortex are necessary for the "image enhancement" of brief acoustic transients (see Buchfellner, Leppelsack, Klump, \& Hausler, 1989).

Three lines of evidence suggest that structures in the visual system more rostral than the brainstem may be similarly important for the inhibitory influence of light pulses on the acoustic startle reflex.

The most dramatic of these, if perhaps the least certain in its implications, is the classic case history provided by Hilgard and Wendt (1933), which describes the effect of localized cortical ablation on reflex modification in a human subject. A patient in the New Haven Hospital had had a cortical tumor removed, which necessitated a complete surgical resection of the left occipital lobe and partial ablation of the left parietal cortex. As a result, she was blind to visual stimuli in the right visual field and she also suffered a more complex "loss of visual memories and marked defects of visual apprehension." For this person, relatively weak light flashes (1-5 fc, approximately) presented in the left visual field had their normal effects on the acoustic blink reflex (which for the human eyeblink is reflex facilitation at a very brief interval and reflex inhibition at a moderately longer interval), but the same stimuli had no effect when presented in the right visual field. A very bright stimulus (" $1,000+\mathrm{fc}$ ") was 
equally effective on both sides, but the authors thought it likely that the intense light had scattered to other parts of the retina and had thus engaged the ipsilateral cortex.

A second indication that the cortex in humans is involved in reflex modification by visual stimuli has been provided by Uhlrich (1983). Uhlrich took advantage of the fact that stereopsis occurs as a result of activity in the binocular cells of the cortex that combine the disparate information from the separate geniculostriate pathways (Barlow, Blakemore, \& Pettigrew, 1967; Ferster, 1981; Poggio \& Fischer, 1977; Poggio \& Talbot, 1981). Thus he proposed that if reflex inhibition could be produced by a dichoptic stereoscopic contour (Julesz, 1960), then cortical activity occasioned by a preliminary stimulus must be at least a sufficient condition for reflex inhibition to occur. Uhlrich (1983) was able to demonstrate that momentary contours produced by a coordinated displacement of a rectangular block within two otherwise random dot matrices, presented separately to the two eyes, did inhibit the reflex blink elicited by a cutaneous stimulus. Appropriate controls showed that drifting monocular stimuli did not have the effect of the binocular stereoscopic patterns.

These findings in human subjects have had some modest further support in the rat. This third indication that structures rostral to the brainstem are involved in reflex inhibition produced by visual stimuli comes from serendipitous findings in the Rochester laboratory some years ago. The experimental series relevant to this point had been focused on the possible contributions of various limbic lesions to reflex inhibition determined by both visual and auditory prestimuli (Kemble \& Ison, 1971). The important outcome of the study at that time was that septal, amydaloid, and hippocampal lesions did not have any effect on startle reflex inhibition caused by prestimuli in either modality. Our minor findings, more relevant to the present concern, were that inhibition produced by visual stimuli was lost in 1 rat with damage to the optic tracts (which is not surprising, of course), and similarly lost in 2 rats with accidental damage to the lateral geniculate nucleus (LGN). The absence of reflex inhibition in the latter two animals indicated that the geniculate pathways are important for reflex inhibition, as Uhlrich (1983) concluded. On the other hand, however, besides the rather adventitious nature of the effects in just 2 animals, it is entirely possible that the lesions of LGN disrupted downward-coursing fibers (e.g., to the colliculi and other brainstem areas; see Ribak \& Peters, 1975) rather than those headed for the cortex. Thus, these data do not necessarily speak to the essential importance of cortical activity to the inhibitory process.

The present data were gathered to determine whether visual stimuli's inhibition of the startle reflex would be sensitive to a massive but temporary dislocation of cortical function in the rat. This was accomplished through the topical application of a solution of $\mathrm{KCl}$ to a small section of cortex-actually to the exposed overlying durafollowing the procedures of Bureš and Burešová (1971).
One advantage of this procedure was that it provided a temporary functional effect that allowed each subject to be used as its own control, in counterbalanced order. The second and more important advantage is that testing could begin as soon as the treatment was imposed, thus obviating the need for a postsurgical recovery time in which subcortical mechanisms might be reorganized and become effective. The effect of a $\mathrm{KCl}$ application is evident within a few minutes as a loss of placing reactions and in the failure of the animal to adjust its posture in response to limb displacement.

\section{METHOD}

\section{Subjects}

The subjects were 10 naive male and female rats, hooded and albino, ranging from 3 to 6 months of age. The animals, raised in the vivarium at the University of Rochester, had been maintained after weaning in individual cages with ad-lib food and water on a 12:12-h light:dark cycle. The experiment was run during the light portion of this cycle. Because of the many reports of retinal damage produced in rats by near normal levels of illumination (see, e.g., Noell, Walker, Kang, \& Berman, 1965), the light level in the colony was kept at a minimal level $(5 \mathrm{fc}$ ) consistent with good animal husbandry. The light level at the back of each empty cage was less than $.5 \mathrm{fc}$.

\section{Apparatus}

The subject was confined for testing in a tunnel-shaped cage $(17 \times 13 \times 9 \mathrm{~cm}$ high) of wire mesh, set on a suspended acrylic platform. A transducer attached to the platform below the cage detected the force of the rat's startle reaction to a sound burst. This assembly was placed in a sound-attenuating chamber $(35 \times 65 \times 43 \mathrm{~cm})$ inside a single-walled, sound-attenuating room $(1.9 \times 2.8 \times 2.5 \mathrm{~m})$ constructed by the Industrial Acoustic Company (Bronx, NY). The startle stimulus was a 115-dB (SPL) noise burst with peak intensity at $16 \mathrm{kHz}$ and an approximately 5 -dB per octave roll-off. It was $25 \mathrm{msec}$ in duration, including 5 -msec rise and decay times. It was provided by a noise generator, shaped by an electronic switch, amplified, and then delivered to a high-frequency speaker through a relay gate. The light source that provided the preliminary stimulus was a voltage-regulated fluorescent lamp (Sylvania F4TS-CWX) with nearly instantaneous rise and decay times. The lamp was placed in a double-glazed box that was well padded with foam, to eliminate the slight noise that was otherwise coincident with light onset. The intensity of the light was $30 \mathrm{fc}$. Responses were recorded and the experiment was controlled by a DEC PDP-8/a computer through a State Systems interface and SUPERSKED software (Snapper, Kadden, \& Inglis, 1982).

\section{Surgical Procedure}

The rats were anesthetized with xylazine $(5 \mathrm{mg} / \mathrm{kg}$ i.m.) and ketamine $(90 \mathrm{mg} / \mathrm{kg}$ i.m.) and placed in a Kopf stereotaxic instrument. A $1-\mathrm{cm}$-long midline incision was made at about bregma, the underlying fascia was cleared away, and then a pair of 2-mm-diameter holes were trephined with their centers $7 \mathrm{~mm}$ posterior to bregma and $5 \mathrm{~mm}$ lateral to the sagittal suture. Great care was taken not to injure the dura. Then, short segments of plastic tubing were cemented in place over the holes and the assembly covered with dental acrylic. The tubes were filled with saline and their tops sealed with stainless steel screws. On test days, the animal was lightly anesthetized by being placed in an ether-charged bell jar. The cannulae were opened and rinsed out, then filled with either a $25 \%$ $\mathrm{KCl}$ solution or a $.9 \%$ saline solution. The examination for loss of motor control began 5-10 min later. The test procedure was to 
place the animal along the edge of a table and gently extend its forelimb and hindlimb so that they hung over the table's edge. The animal treated with saline immediately and invariably pulled both limbs back up toward its body and placed them on the table top, whereas following $\mathrm{KCl}$ treatment the limbs were left loosely extended. At the end of the test session, the postural test for behavioral decortication was repeated. The animal was lightly anesthetized again, and the cannulae were rinsed and then filled with saline.

\section{Behavioral Procedure}

Behavioral testing began about $.5 \mathrm{~h}$ after the $\mathrm{KCl}$ or saline treatment, including a 15 -min interval spent in the dark testing cage. Each of the testing series began with 4 warm-up trials delivered at 30-sec intervals, given in order to set the amplifier gain on the response recorder and to allow the startle response to stabilize, because the first several trials after an extended quiet period tend to be unusually large. The trials within the experimental series began immediately thereafter. There was a total of 90 trials, on average 30-sec apart. Ten control (startle-alone trials) were interspersed with 10 trials in each of eight conditions in which the startle stimulus was preceded by the light flash at onset-to-onset intervals of 20 , $40,70,110,160,220,320$, and $500 \mathrm{msec}$. The order of trials was random, determined by the computer with the constraint that no condition was repeated until all had been presented for an equal number of trials. All of the rats were tested twice, randomly beginning with the $\mathrm{KCl}$ treatment $(n=6)$ or with saline $(n=4)$. The second test was given from 1 to 8 days after the 1 st $(M=3.9$ days).

\section{RESULTS}

In Figure 1 are described the mean relative response amplitudes, and their standard errors, for all interstimulus interval (ISI) conditions in both cortical states. The transformed data shown in this graph began with the mean response amplitudes for each condition for each separate subject in each separate brain state, these means then expressed as a proportion of that subject's mean baseline control response (with the startle stimulus alone, by definition, equal to $100 \%$ ) in the same state. It is apparent

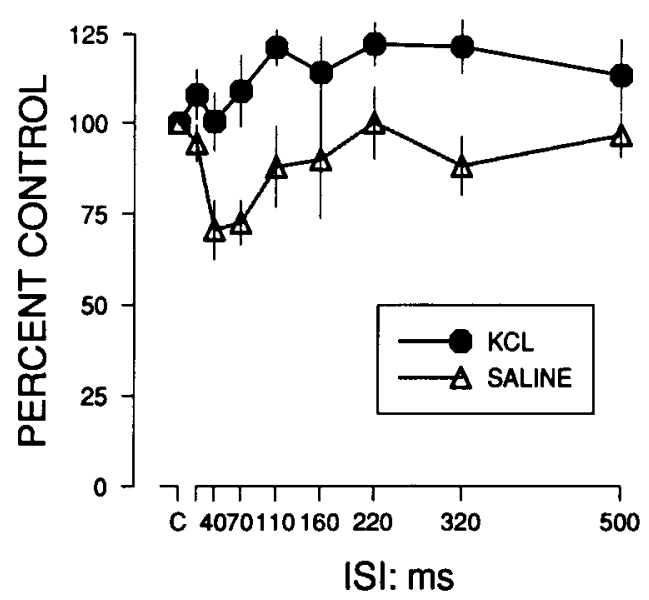

Figure 1. Mean relative response amplitudes $( \pm S E M)$ when the startle reaction is preceded by a 20 -msec light flash at various intervals (20-500 msec), expressed as a proportion of the startlestimulus-alone control baseline (at $C$ ), after topical $\mathrm{KCl}$ or saline had been applied to the cortex of the rat. in Figure 1 that the two cortical treatments had substantially different effects on reflex modification, and that these differences were present, to various degrees, across the range of ISIs. Following the application of saline to the cortex, the light inhibited the startle reflex, primarily at the 40- and 70-msec intervals. This is the standard finding that we obtain in normal intact rats in this paradigm. The data obtained following the application of $\mathrm{KCl}$ to the cortex stand in sharp contrast to the standard outcome. Now the light provided no hint of an effect at the intervals for which inhibition normally occurs, but, instead, actually facilitated the response at the longer intervals of $110 \mathrm{msec}$ and above. Analysis of variance (ANOVA) of the data presented in Figure 1 produced a significant difference between the overall relative response values of the saline versus the $\mathrm{KCl}$ applications $[F(1,9)=13.84$, $p<.01]$. The effect of ISI was modestly significant $[F(8,72)=2.18, p<.05]$, as was the interaction between the $\mathrm{KCl}$ versus saline condition and the quartic trend for the ISI function $[F(1,9)=9.31, p<.02]$. Similar results were obtained in the ANOVA of the absolute response amplitudes (measured in volts). As we have found in other work (Ison et al., 1991), the KCl treatment had a varied effect on baseline startle amplitudes, with some animals showing a severe loss of responsivity (5 of the animals had their control values reduced by $50 \%$ or more) while the others showed essentially no effect. While the group mean in the no-light control condition after saline treatment was reduced by one third after the $\mathrm{KCl}$ application, as a result of the large individual differences the main effect for the $\mathrm{KCl}$ versus saline treatment did not reach the standard level of significance for the group taken as a whole $[F(1,9)=3.58, p<.10]$, despite the reliable decrement in startle behavior obtained in certain animals. The effect of ISI in the absolute response data appeared in the quartic trend component $[F(1,9)=8.12$, $p<.02]$, and this trend component was affected by the cortical manipulation $[F(1,9)=6.02, p<.05]$. There was no suggestion in these data that the variable effect of $\mathrm{KCl}$ in reducing the control level of startle responding was at all related to its more uniform influence in diminishing the inhibitory effect of the light flash on startle behavior. In contrast to the substantial individual differences in susceptibility to $\mathrm{KCl}$ noted for reflex elicitation, all animals showed the loss of inhibition following $\mathrm{KCl}$ at the brief ISIs, and all showed the presence of facilitation at the long intervals. It is of further interest that the correlation between changes in baseline reflex elicitation and the changes in reflex modification produced by $\mathrm{KCl}$ was about zero $(r=.06)$.

Turning to the ANOVA of the two cortical states considered separately, within the $\mathrm{KCl}$ condition the increase in reflex strength at the longer lead times produced a significant quadratic trend for ISI on relative amplitudes $[F(1,9)=7.68, p<.05]$, whereas within the saline condition the decrement in reflex strength at the shorter lead times produced a significant quartic trend for ISI $[F(1,9)=7.43, p<.05]$. The same two trend effects 
were significant for the absolute response values as well, and the main effect of ISI was also significant for both the $\mathrm{KCl}$ and the saline conditions in these absolute response data $(p<.05)$. These analyses attest to the reliability of both the inhibitory effect of the light in the normal cortical state and its contrary facilitative effect when the cortex was depressed, and show further the differences in the temporal course of these two processes.

\section{DISCUSSION}

The empirical consequence of functional decortication for reflex inhibition by visual stimuli may be readily described, as can some aspects of its theoretical significance; but other implications of these data are more provocative than certain and should serve, therefore, as the impetus to a more detailed dissection of the contributions of forebrain structures to reflex modification rather than as conclusions. The data are clear in showing, first, that the relatively early inhibitory effect of a brief light flash on the acoustic startle reflex is lost with functional decortication, and second, that at the longer lead times at which the light flash appears to be approximately neutral in its effect under normal conditions, the light serves to facilitate the startle reflex for the functionally decorticate animal. As we have found in other experiments (Ison et al., 1991), a common, but not invariable, effect of functional decortication is to depress the control startle reflex, substantially in some animals, and very little or not at all in others. The reasons for its differential effectiveness across animals is not evident to us. It is clear, however, that animals tend to be limp and flaccid after the $\mathrm{KCl}$ treatment, and it is reasonable to think that this condition of minimal muscular tonus would reduce the normally facilitative neuromuscular background for the motor response.

At first glance, it will be thought that the loss of lightinduced inhibition of the startle reflex might be secondary to a "floor effect," following the reduction in control startle amplitudes by $\mathrm{KCl}$. Contradicting this, we note that the remaining control response was substantial in every animal, and also that there was no indication that the changes in reflex modification produced by $\mathrm{KCl}$ were at all related to its effect on reflex elicitation. Another possible explanation of the effect of $\mathrm{KCl}$ application on reflex inhibition is similarly artifactual in nature-that is, that the decorticate animal closes its eyes in the test chamber and thus never is really presented with the light flash. In the early stages of the present research, this possibility was a matter of some concern, and so the functionally decorticate rats were monitored after they were placed in a dim red light. These animals were very quiet under these conditions, but their eyes remained open. But in addition to these initial observations, we can now safely assert that the visual stimulus had been received and processed following the $\mathrm{KCl}$ application-but processed in some abnormal way, because of the reliable facilita- tion provided by the visual input at the longer lead times that are normally of little consequence for reflex behavior.

The loss of inhibition following the $\mathrm{KCl}$ applications admits of several different explanations, the most simple being that, in consistency with the prior reports, activity in visual cortex is certainly a sufficient precursor to reflex inhibition produced by visual stimuli (Uhlrich, 1983), at least given the integrity of brainstem structures (Leitner \& Cohen, 1985), and it may perhaps even be a necessary precondition (Hilgard \& Wendt, 1933). In this respect, light prepulses are different from noise prepulses, which exert their influence in the absence of normal cortical function and must be presumed to depend more simply on brainstem mechanisms for their effect (Davis \& Gendelman, 1977; Ison et al., 1991).

However, although this is one reasonable explanation of the effect of functional decortication on reflex inhibition, it is not the only one. These other hypotheses should also be considered, especially because some other results may in fact contradict it-specifically, Davis (personal communication) has seen normal light-produced inhibition in rats following the bilateral removal of the occipital poles, and, presumably, the primary visual cortex. To be sure, the limits of primary visual cortex may not be certain in the rat, and there are other ways in which visual input can get to other visual cortical areas; nonetheless, these data appear to deny any simple role for primary visual cortex in the expression of reflex inhibition in the rat. Of still further interest, Davis (personal communication) has found that lesions of the perirhinal cortex (which receives information from both cortical and subcortical visual areas) totally block visual prepulse inhibition at short ISIs. These recent studies have only involved animals conditioned to fear the visual stimulus, which normally show prepulse inhibition at short intervals and then a learned facilitation at intervals at which the light has been paired with a shock (see, e.g., Davis, Schlesinger, \& Sorenson, 1989); but there is no reason to suspect that the inhibitory effect obtained in these experiments is different from that which has captured our interest. With respect to these observations from the Davis laboratory, it should be remembered that the $\mathrm{KCl}$ application renders the entire cortex dysfunctional, not simply the primary visual cortex; thus, it may be quite reasonable to conclude that visual input must be processed in some other cortical area, such as, for example, the perirhinal area, in order for that stimulus to engage the brainstem inhibitory areas.

But finally, as a last alternative, it is known that although cortical depression does not spread directly to subcortical structures, it certainly affects their normal function, presumably by withdrawing a source of normal modulatory input. Thus, as summarized by Bures, Buresova, and Krivanek (1974, pp. 172-184), cortical spreading depression may alter both the background firing rates and evoked neuronal responses in the thalamus, hypothalamus, caudate nuclei, and reticular formation, although short-latency evoked responses in the primary 
sensory pathways appear to be normal. It is therefore not unreasonable to hypothesize that the destructive consequence of functional decortication on reflex inhibition by visual stimuli and its promotion of their facilitative effects may both arise because of its indirect influence on essential subcortical centers. In fact, this hypothesis might be appropriately applied also to the effects noted by Hilgard and Wendt (1933), not only because the surgical resection extended beyond the primary visual cortex, but also because the tumor itself may have had broader ramifications for cortical function.

In conclusion, it is now certain that normal cortical function is a necessary prerequisite if light-pulse prestimuli are to have their normal inhibitory effect on the acoustic startle reflex in the rat. It is also certain, from our earlier reports, that normal cortical function is not a necessary prerequisite for sound pulses to inhibit the acoustic startle reflex. Whether this difference results directly because visual cortex is essential to light-pulse inhibition while auditory cortex is not important for sound-pulse inhibition, or whether other cortical areas control subcortical mechanisms important for light-prepulse inhibition, but not for sound-prepulse inhibition, are hypotheses that must be tested in subsequent research.

\section{REFERENCES}

Barlow, H. B., Blakemore, C., \& Pettigrew, J. D. (1967). The neural mechanisms of binocular depth perception. Journal of Physiology, 193, 327-342.

Buchfellner, E., Leppelsack, H.-J., Klump, G. M., \& Hausler, U. (1989). Gap detection in the starling (Sternus vulgaris): II. Coding of gaps by forebrain neurons. Journal of Comparative Physiology $A$, $164,539-549$

BUREš, J., \& BUREŠová, O. (1971). Inducing cortical spreading depression. In R. D. Myers (Ed.), Methods in psychobiology (Vol. 2, pp. 319-343). New York: Academic Press.

Bureš, J., \& Burešoví, O., \& Krivanex, J. (1974). The mechanism of applications of Leao's spreading depression of electroencephalographic activity. New York: Academic Press.

Davis, M., \& Gendelman, P. M. (1977). Plasticity of the acoustic startle reflex in acutely decerebrate rats. Journal of Comparative \& Physiological Psychology, 91, 549-563.

Davis, M., SChlesinger, L. S., \& Sorenson, C. A. (1989). Temporal specificity of fear conditioning: Effects of different conditioned stimulusunconditioned stimulus intervals on the fear-potentiated startle. Journal of Experimental Psychology: Animal Behavior Processes, 15, 295-310.

FERSTER, D. (1981). A comparison of binocular depth mechanisms in areas 17 and 18 of the cat visual cortex. Journal of Physiology, 315, 623-655.
Hammond, G. R. (1974). Frontal cortical lesions and prestimulus inhibition of the rat's acoustic startle reaction. Physiological Psychology, 2, 151-156.

Hilgard, E. R., WEndT, G. R. (1933). The problem of reflex sensitivity to light studied in a case of hemianopsia. Yale Journal of Biology \& Medicine, 5, 373-385.

Hoffman, H. S., \& Ison, J. R. (1980). Reflex modification in the domain of startle: I. Some empirical findings and their implications for how the nervous system processes sensory input. Psychological Review, 87, 175-189.

Ison, J. R., O'Connor, K., Bowen, G. P., \& Bocirnea, A. (1991). Temporal resolution of gaps in noise by the rat is lost with functional decortication. Behavioral Neuroscience, 105, 33-40.

JuLESz, B. (1960). Binocular depth perception of computer-generated patterns. Bell System Technical Journal, 39, 1125-1162.

Kemble, E. J., \& IsoN, J. R. (1971). Limbic lesions and the inhibition of startle reactions in the rat by conditions of preliminary stimulation. Physiology \& Behavior, 7, 925-928.

Leitner, D. S., \& Cohen, M. E. (1985). The role of the inferior colliculus in inhibition of acoustic startle in the rat. Physiology \& Behavior, 34, 65-70.

Leitner, D. S., Powers, A. S., Stitt, C. L., Hoffman, H. S. (1981). Midbrain reticular formation involvement in the inhibition of acoustic startle. Physiology \& Behavior, 26, 259-268.

Noell, W. K., Walker, V. S., Kang, B. S., \& Berman, S. (1965). Retinal damage by light in rats. Investigative Ophthalmology, 5, 450-473.

Poggio, G. F., \& Fischer, B. (1977). Binocular interaction and depth sensitivity in the striate and prestriate cortex of the behaving monkey. Journal of Neurophysiology, 40, 1392-1405.

Poggio, G. F., \& TAlbot, W. H. (1981). Mechanisms of static and dynamic stereopsis in foveal cortex of the rhesus monkey. Journal of Physiology, 315, 469-492.

RiBak, C. E., \& PETERs, A. (1975). An autoradiographic study of the projections from the lateral geniculate body of the rat. Brain Research, 92, 341-368.

Saitoh, K., Tilson, H. A., Shaw, S., \& Dyer, R. S. (1987). Possible role of the brainstem in the mediation of prepulse inhibition in the rat. Neuroscience Newsletters, 7, 216-222.

SeChenov, I. M. (1965). Reflexes of the brain (S. Belsky, Trans.). Cambridge, MA: MIT Press. (Original work published in St. Petersburg: Sushchinski, 1863)

SNAPPER, A. G., KadDen, R. M., \& INGLis, G. B. (1982). State notation of behavioral procedures. Behavioral Research Methods \& Instrumentation, 14, 329-342.

UHLRCH, D. J. (1983). Inhibition of the human blink reflex by visual form stimuli. Unpublished doctoral dissertation, Brown University, Providence, RI.

YouNG, J. S., \& FECHTER, L. D. (1983). Reflex inhibition procedures for animal audiometry: A technique for assessing ototoxicity. Journal of the Acoustical Society of America, 73, 1686-1693.

(Manuscript received January 23, 1991; revision accepted for publication March 20, 1991.) 